\title{
Effect of Faraday mirror imperfections in a fiber optic current sensor dedicated to ITER
}

\author{
Doğuş Karabulut $^{\mathrm{a}, \mathrm{b}}$, Anton Miazin ${ }^{\mathrm{b}, \mathrm{c}}$, Andrei Gusarov ${ }^{\mathrm{c}}$, Philippe Moreau ${ }^{\mathrm{d}}$, Willem Leysen ${ }^{\mathrm{c}}$, \\ Patrice Mégret $^{\mathrm{b}}$, Marc Wuilpart ${ }^{\mathrm{b}, *}$ \\ ${ }^{a}$ Electrical and Electronics Engineering Department, İzmir Institute of Technology, Gülbahçe Mah., 35430 Urla, İzmir, Turkey \\ ${ }^{\mathrm{b}}$ Department of Electromagnetism and Telecommunications, University of Mons, Boulevard Dolez 31, 7000 Mons, Belgium

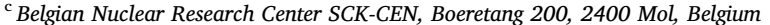 \\ ${ }^{\mathrm{d}}$ CEA Cadarache (IRFM), 13115 Saint-Paul-lez-Durance, France
}

\section{A R T I C L E I N F O}

\section{Keywords:}

Fiber optics current sensors

Polarimetric sensors

Plasma diagnosis

\begin{abstract}
A B S T R A C T
Plasma current measurements in ITER are safety-related and must therefore satisfy a very demanding specification. In this paper, the use of the Fiber Optics Current Sensor (FOCS) operating in the reflection mode with a Faraday mirror to perform plasma current measurements is analyzed. Based on the Jones matrix formalism, we performed numerical simulations to investigate the impact of the Faraday mirror detuning on the measurement accuracy. We show that the use of standard commercial components does not allow to satisfy the ITER requirements for the whole plasma current range. A simple solution to the problem is proposed, which consists in taking into account a mirror calibration in the current estimator. We show that the achievable mirror calibration accuracy is sufficient to fulfill the ITER requirements.
\end{abstract}

\section{Introduction}

An accurate measurement of plasma current is essential for a safe control of a Tokamak. Nowadays, these measurements are performed using various types of inductive sensors such as Rogowski or Pick-up coils $[1,2]$. The signal of such sensors is proportional to the time derivative of the magnetic flux through the loop and to find the current, a time-integration step is required. However, in future burning plasma installations, ITER and later in DEMO, the presence of strong radiations combined with steady-state operation will create a difficult problem when operating with such inductive sensors: during steady-state plasma operation, the signal will be strongly perturbed by the radiation-induced noise and time-integration may result in a significant drift $[3,4]$. To overcome this problem, two types of steady-state sensors placed outside the vacuum vessel are included in the ITER magnetic diagnostics system: Hall sensors and FOCS (Fiber Optics Current Sensor) [5]. Hall sensors based systems will provide local measurements of the tangential and normal components of the magnetic field [6]. In ITER, three poloidal arrays of 60 sensors will be welded to the vacuum vessel outer skin and distributed toroidally in three vacuum vessel sections. The plasma current is obtained by integrating the magnetic field all along the contour defined by all the probes. In a short term, the accuracy of such a measurement requires the accurate calibration of the 120
Hall probes (60 normal field and 60 tangential field sensors). Maintaining a very accurate measurement capability over the whole ITER life-time is required. This is a challenging task because the Hall sensors will be exposed to high levels of nuclear radiation and could not be replaceable. The radiation-induced noise in the long cables connecting the sensor head and the electronic system requires additional attention.

FOCS systems are based on the Faraday rotation of the state of polarization (SOP) experienced by a lightwave propagating through the sensing fiber that encircles the current. The Faraday rotation is directly proportional to the integral magnetic field component aligned with the sensing fiber. As a consequence, no time integral is required, and the plasma current is directly obtained from the sensor output. Moreover, the sensor is not sensitive to radiation-induced currents and, in contrast to Hall sensors, the fiber used in the ITER FOCS system is replaceable [7].

The Faraday effect is also the basis for the free space rotation-based laser polarimetry used to obtain data on the plasma current spatial density profile [8]. Such a system is installed on the EAST tokamak, where it provides important current profile information to support the plasma control [9]. To perform measurements, special precautions are required to reduce the measurement accuracy degradation due to misalignments, interference from multiple reflections when the plasma density varies with time, and stray light coming from other sources.

\footnotetext{
* Corresponding author.

E-mail address: marc.wuilpart@umons.ac.be (M. Wuilpart).
} 
Table 1

Relevant ITER specifications [1].

$\begin{array}{ll}\text { Plasma current measurement accuracy } & \\ \text { High range [1-17 MA] } & \text { Relative accuracy of \%1 } \\ \text { Low range [0-1 MA] } & \text { Absolute accuracy of } 10 \mathrm{kA}\end{array}$

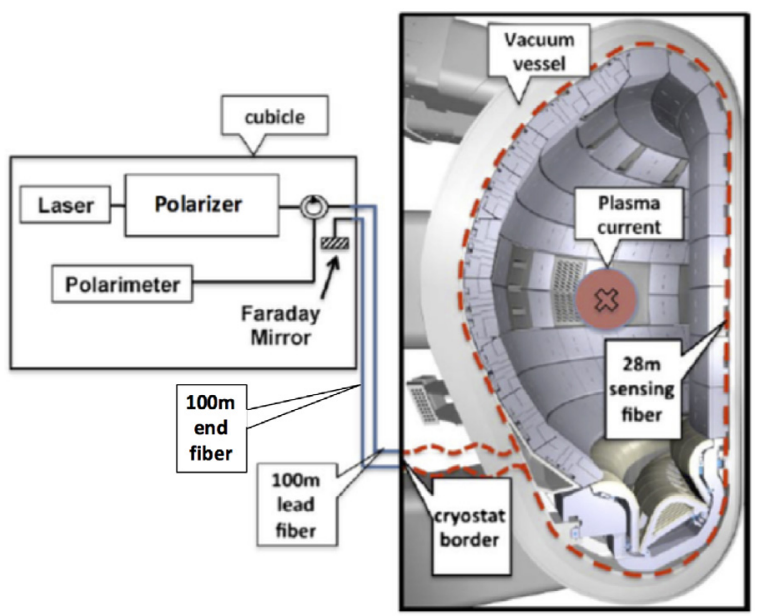

Fig. 1. FOCS implementation for plasma current in ITER.

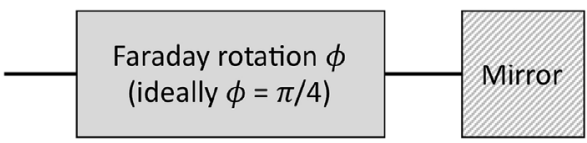

Fig. 2. Faraday mirror modelling.

Misalignment and stray light do not perturb the performance of FOCS, but there are other parasitic effects which influence its operation. An important problem is the presence of the linear birefringence, which originates from both fiber intrinsic properties and external mechanical perturbations $[10,11]$. In order to alleviate these detrimental effects, the use of the FOCS operating in the reflection mode with a Faraday mirror has been proposed [12-14]. A Faraday mirror reflects light and rotates the SOP with a total angle of $90 \mathrm{deg}$ in order to exchange the $x$ and $y$ components of the lightwave. In the absence of magnetic field, this approach allows the full compensation of the intrinsic birefringence effect. However, in the presence of the non-reciprocal birefringence induced by the magnetic field, the full compensation is not possible [15].

Recent works published by the authors $[16,17]$ demonstrated that when the FOCS uses a spun fiber with adequate parameters, the ITER specifications regarding the required plasma current measurement accuracy (see Table 1 [1]) can be fulfilled. A spun fiber is characterized by some intrinsic linear birefringence quantified by its beat length $L_{B}$ [26] and by a rotation of its linear birefringence axis along the fiber length. The rotation is quantified by the spun period $S_{P}$ representing the length after which the birefringence axis has completed a $2 \pi$ rotation. Note that the merit of using spun fibers for current measurement $[12,18]$ lies in the fast rotation (small $S_{P}$ ) of the birefringence axis along the fiber without generation of shear stresses, so that the detrimental effect of the intrinsic birefringence is greatly reduced. In [16], it was shown that a $L_{B} / S_{P}$ ratio larger than 10.14 allows to satisfy the ITER requirements. However, that study did not include temperature effects that modify both the fiber Verdet constant and the linear birefringence. The inclusion of temperature constraints [17] led to a corrected minimum $L_{B} / S_{P}$ ratio equal to 19.20. In our previous works, the ITER relevant FOCS setup shown in Fig. 1 was analyzed. A laser launches light with a linear SOP via a polarizer and a circulator into the FOCS fiber. The fiber consists of three parts: (1) $\mathrm{a} \simeq 100 \mathrm{~m}$ long lead fiber connecting the
FOCS data acquisition system placed in the cubicle area to the sensing fiber, (2) a $\simeq 28 \mathrm{~m}$ long sensing fiber making a loop around the current (around the ITER vacuum vessel) and (3) an end fiber of $100 \mathrm{~m}$ connecting the end of the sensing fiber to the Faraday mirror installed in the cubicle. A polarimeter is used to measure the SOP rotation induced by the plasma current after a round-trip propagation. The study detailed in this paper is related to the same FOCS setup.

Our previous studies considered the Faraday mirror as a perfect one: the roundtrip rotation induced by the component is exactly equal to 90 deg. However, in a FOCS using a real Faraday mirror, the rotation does not exactly match $90 \mathrm{deg}$ and the intrinsic linear birefringence is compensated less efficiently. The purpose of this paper is to study by means of numerical simulations the influence of the Faraday mirror rotation detuning on the ITER FOCS plasma current measurement accuracy and to propose a solution to reduce its detrimental effect. The results show that in the case of ITER, neglecting the Faraday mirror detuning may result in unacceptable errors. We also propose a simple solution to this problem and quantify the corresponding error reduction.

\section{Principles of FOCS}

The principle of FOCS is described in many publications, for example, see [19]. When an optical fiber encircles a current (as in the setup shown in Fig. 1), a circular birefringence is induced in the fiber resulting in a rotation of the SOP along the fiber loop. Since the light beam passes twice in the loop (after reflecting from the Faraday mirror) and the Faraday mirror adds a $90^{\circ}$ extra rotation, the plasma current is given by [16]:

$I_{P}=\frac{\theta_{T}-\pi / 2}{2 V \mu_{0}}$.

where $\theta_{T}$ is the measured SOP rotation and $V$ is the Verdet constant. For standard silica fibers, the Verdet constant is equal to $0.54 \mathrm{rad} \cdot(\mathrm{m} \cdot \mathrm{T})^{-1}$ for a wavelength of $1550 \mathrm{~nm}$ [20].

In a real optical fiber, an additional intrinsic or extrinsic birefringence is present. This birefringence causes an unwanted extra SOP modification and a change of the azimuth and the ellipticity. The presence of a linear birefringence together with the use of a non-perfect Faraday mirror contributes to a measurement error when using Eq. (1). The detrimental effect of the intrinsic birefringence has already been investigated $[16,17]$. In order to take into account the Faraday mirror detuning, the modeling proposed in [16] has been extended as described in the next section.

\section{Optical fiber modeling}

The fiber modeling approach is identical to that presented in [16]: the fiber is modeled as a stack of $N$ small sections of length $l=\min \left(L_{B}\right.$, $S_{P}$ )/100 over which the polarization properties are assumed to be uniform and defined by its local Jones matrix $J_{i}$. The complete expression of the Jones matrices can be found in [16]. To validate this approach, we compared the Jones matrices of a spun fiber obtained from the stack modeling with the Jones matrices obtained from the analytical expression found in reference [12]. The spun fiber characteristics were taken relevant to our case $\left(L_{B}=57.6 \mathrm{~mm}\right.$ and $S_{P}=3 \mathrm{~mm}$, see Section 4) and the fiber length varied in a range from $0.03 \mathrm{~mm}$ to $28 \mathrm{~m}$ (length of the sensing fiber in ITER) with a step of $0.03 \mathrm{~mm}$. The difference between the output normalized Stokes parameters obtained with the modeling and the analytical solution stayed below 0.052 .

It may be useful to note that the linear birefringence is considered identical for each section and is given by $\delta=2 \pi / L_{B}$ while the linear birefringence axis rotates with a period $S_{P}$. The circular birefringence induced by the magnetic field is also considered constant and is given 

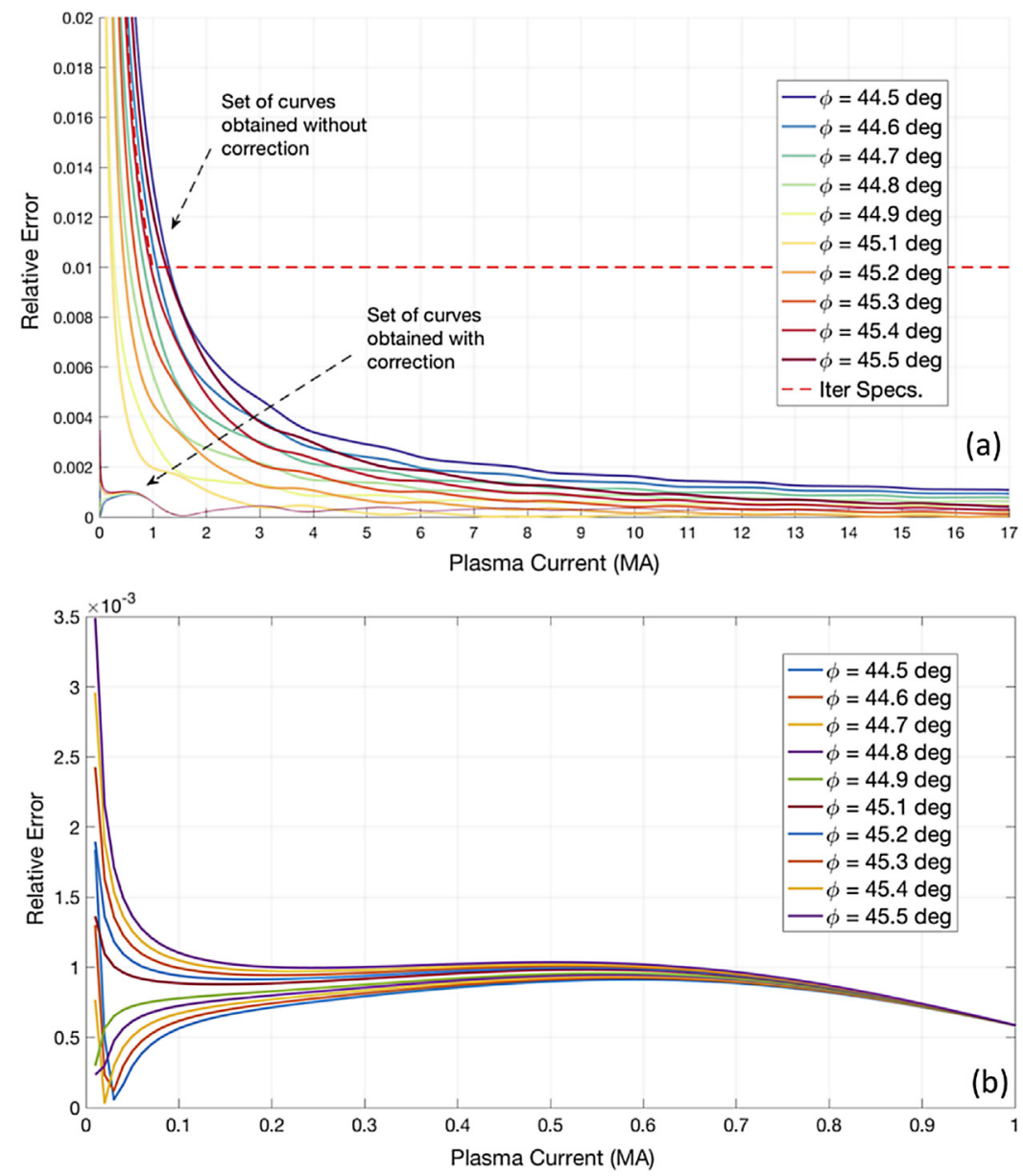

Fig. 3. (a) Relative errors obtained without and with correction of equation (1). (b) Zoom on the relative error obtained with correction for $0<I_{P}<1$ MA.

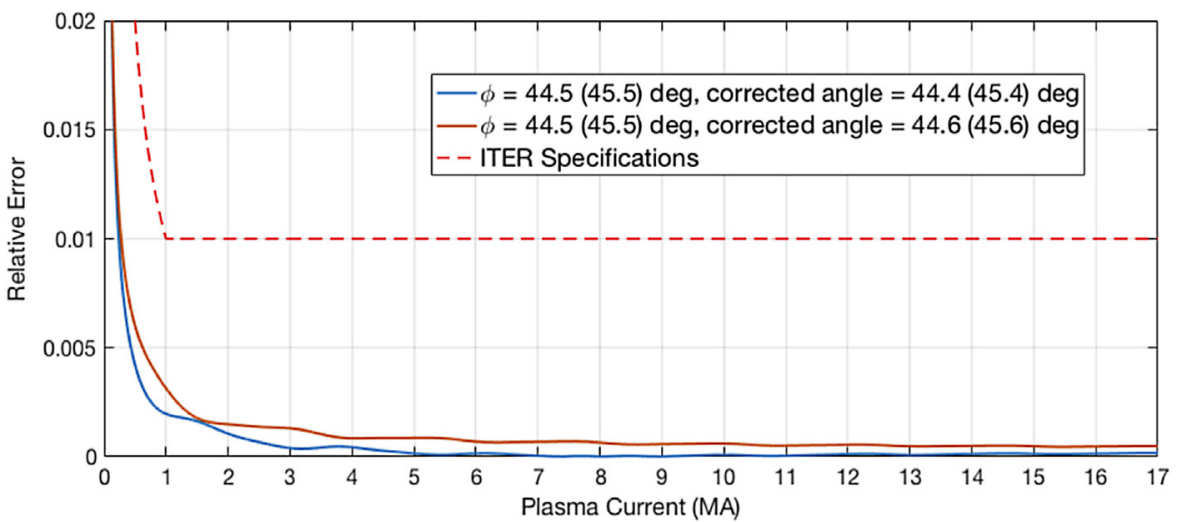

Fig. 4. Relative error on plasma current measurement for $\phi=45.5 \mathrm{deg}$.

by $\rho=V B$ where $B=\mu_{0} I_{P} /(2 \pi R), R$ being the average radius of the vacuum vessel. We can assume a circular shape of the vacuum vessel because it has been shown in [23] that the performance of FOCS is only slightly dependent on the distribution of the magnetic field along the fiber (the D-shape of the ITER configuration is therefore not modeled). Taking into account the roundtrip propagation, the output Jones vector $\boldsymbol{V}_{\text {out }}$ seen by the polarimeter is calculated as $[16,24]$ :

$\boldsymbol{V}_{\text {out }}=J_{1} \ldots J_{i} \ldots J_{N-1} J_{N} J_{\mathrm{FM}} J_{N} J_{N-1} \ldots J_{i} \ldots J_{1} \cdot \boldsymbol{V}_{\text {in }}$,

where $J_{\mathrm{FM}}$ represents the Jones matrix of the Faraday Mirror. Ideally, the Faraday mirror consists of a non reciprocal $45 \mathrm{deg}$ rotator followed by a classical mirror as shown in Fig. 2. The Faraday mirror Jones matrix $J_{\mathrm{FM}}$ therefore corresponds to a concatenation of two $45^{\circ}$ rotators with a classical mirror in between [13]. However, for a real Faraday mirror, the rotation angle can differ from 45deg (rotation angle detuning). Taking this into account, an extra parameter $\phi$ (the Faraday mirror rotation angle), was added in the modeling, as compared to the model implemented in [16]. The Faraday mirror Jones matrix is calculated as:

$J_{\mathrm{FM}}=\left[\begin{array}{cc}\cos \phi & -\sin \phi \\ \sin \phi & \cos \phi\end{array}\right]\left[\begin{array}{ll}1 & 0 \\ 0 & 1\end{array}\right]\left[\begin{array}{cc}\cos \phi & -\sin \phi \\ \sin \phi & \cos \phi\end{array}\right]=\left[\begin{array}{cc}\cos 2 \phi & -\sin 2 \phi \\ \sin 2 \phi & \cos 2 \phi\end{array}\right]$.

The unit matrix in Eq. (3) is the matrix of a conventional mirror, which is true when considering unchanged $x$ and $y$ axes for the two 
propagation directions [26]. When $\phi=45 \mathrm{deg}$, the ideal Faraday mirror matrix is obtained. Commercial products usually have a Faraday rotation uncertainty of $\pm 0.5 \mathrm{deg}$ for $45 \mathrm{deg}$ rotation (44.5 deg $<\phi$ $<45.5 \mathrm{deg}$ ), for example see [25]. This uncertainty is considered in the simulations.

The length of the leading fiber $(\simeq 100 \mathrm{~m})$ is chosen in a way that the SOP at the input of the sensing fiber is the furthest from a linear state (largest ellipticity) such as the leading fiber length corresponds to a worst-case scenario as explained in [16]. In [16], it is indeed demonstrated that the measurement error increases if the SOP ellipticity at the sensing fiber input gets larger.

The relative plasma current error is calculated by comparing the plasma current determined by the FOCS system, i.e. obtained by using Eqs. (1), (2) and (3), with the value which was used to define the magnetic field.

\section{Results and discussion}

The fiber considered in the simulation is a uniform spun fiber characterized by $L_{B}=57.6 \mathrm{~mm}$ and $S_{P}=3 \mathrm{~mm}$ so that $L_{B} / S_{P}=19.20$. It is indeed demonstrated in [17] that 19.20 is the minimum ratio acceptable for $L_{B} / S_{P}$ that fulfills the ITER requirements when considering the detrimental temperature effects and for an ideal Faraday mirror ( $\phi=45 \mathrm{deg}$ ). For the results presented further in this section, $L_{B}$ and $S_{P}$ were kept unchanged, only the Faraday mirror rotation angle $\phi$ varies.

The set of curves denoted as "Set of curves obtained without correction" presented in Fig. 3(a) shows the evolution of the relative current measurement error with respect to $I_{P}$ for some values of $\phi$. As expected, for a fixed $I_{P}$, the error increases as $\phi$ deviates from $45 \mathrm{deg}$. The dashed line in Fig. 3(a) represents the ITER specifications (see Table 1), which are fulfilled only when $44.70<\phi<45.30 \mathrm{deg}$. This result defines the acceptable uncertainty on the Faraday mirror rotation angle.

When looking at the specifications of commercially-available Faraday mirrors, it appears that such an uncertainty is not provided. According to our simulation results, it is therefore not guaranteed that the ITER specifications will be fulfilled when using a commerciallyavailable Faraday mirror.

An intuitive way to improve the measurement accuracy is to take the deviation into account and to calculate $I_{P}$ from a corrected version of Eq. (1), where $\frac{\pi}{2}$ is replaced by $2 \phi$ i.e. the actual Faraday mirror rotation angle:

$I_{P}=\frac{\theta_{T}-2 \phi}{2 V \mu_{0}}$.

We have performed simulations using this adjusted formula. The obtained results are shown in Fig. 3(a) where they are compared with the uncorrected case. It clearly appears that all the obtained curves are now far below the ITER specifications, bringing a clear improvement of the plasma current estimation accuracy. The curves obtained with the adjusted equation for $0<I_{P}<1 \mathrm{MA}$ are displayed separately in Fig. 3(b). Let us note that the curves obtained with correction are not identical to those obtained for an ideal Faraday mirror since the linear birefringence of the fiber sections (lead, sensing and end fibers) are less efficiently compensated because of the Faraday mirror detuning.

The use of Eq. (4) assumes that the exact value of $\phi$ is known. In practice it is possible to measure the detuning angle only with some accuracy. For example, by using the technique proposed in [27], a precision of \pm 0.1 deg can be achieved. In that method, $\phi$ is determined by using an optical vector analyzer. A highly birefringent fiber is placed between the analyzer and the Faraday mirror under test and the global round-trip polarization mode dispersion (PMD) is measured. The measured PMD is an indicator of the Faraday rotation angle detuning. In order to demonstrate the effect of this 0.1 deg uncertainty, Fig. 4 shows the relative error obtained in the extreme cases i.e. for $\phi=44.5(45.5) \mathrm{deg}$ when using $44.4(45.4)$ and $44.6(45.6) \mathrm{deg}$ as the corrected angle in Eq. (4) to calculate $I_{P}$. It appears that all the curves satisfy the ITER requirements. A $0.1 \mathrm{deg}$ accuracy is therefore enough for the application. Let us note that in Fig. 4, the curve obtained for $\phi=44.5 \mathrm{deg}$ and a corrected angle of 44.4 (44.6) deg is identical to that obtained for $\phi=45.5 \mathrm{deg}$ and a corrected angle of 45.4 (45.6) deg.

\section{Conclusion}

Fiber Optics Current Sensor (FOCS) is considered for installation at ITER to perform plasma current measurements during steady-state operation. This diagnosis is safety-related and the measurement accuracy must satisfy the ITER requirements. In this paper, we studied the influence of the Faraday mirror imperfections on the performance of the ITER FOCS operating in the reflection configuration with a Faraday mirror. Our results indicate that care should be taken when dealing with commercially available components. Their standard rotation uncertainty exceeds the maximum acceptable value allowing achieving the required measurement accuracy. A way to bring the measurement error down to the tolerance limit is to take into account the Faraday mirror rotation detuning in the plasma current calculation. Combined with the Faraday mirror calibration, this approach allows fulfilling the ITER specifications.

\section{Acknowledgments}

This work has been carried out under support from the Belgian Federal Government, also within the framework of the EUROfusion Consortium and has received funding from the Euratom research and training programme 2014-2018 under Grant Agreement No. 633053. The views and opinions expressed herein do not necessarily reflect those of the European Commission, the Belgian Federal Government.

\section{References}

[1] A.J.H. Donné, A.E. Costley, R. Barnsley, H. Bindslev, R. Boivin, G. Conway, R. Fisher, R. Giannella, H. Hartfuss, M.G. von Hellermann, E. Hodgson, L.C. Ingesson, K. Itami, D. Johnson, Y. Kawano, T. Kondoh, A. Krasilnikov, Y. Kusama, A. Litnovsky, P. Lotte, P. Nielsen, T. Nishitani, F. Orsitto, B.J. Peterson, G. Razdobarin, J. Sanchez, M. Sasao, T. Sugie, G. Vayakis, V. Voitsenya, K. Vukolov, C. Walker, K. Young, Chapter 7: Diagnostics, Nucl. Fusion 47 (2007) S337-S384.

[2] Q. Chen, H.-b. Li, B.-X. Huang, Q.-q. Dou, Rogowski sensor for plasma current measurement in J-TEXT, IEEE Sens. J. 9 (2009) 293-296.

[3] E.R. Hodgson, General radiation problems for insulating materials in future fusion devices, J. Nucl. Mater. 258 (1998) 226-233.

[4] P. Moreau, I. Bolshakova, B. Brichard, G. Chitarin, R. Delogu, I. Duran, A. Encheva, Y. Fournier, A. Galo, A. Le-Luyer, J.B. Lister, P. Malard, J.M. Moret, P. Pastor, S. Peruzzo, J. Romero, D. Testa, M. Toussaint, G. Vayakis, R. Vila, Development of a magnetic diagnostic suitable for the ITER radiation environment, Proc. Conference on Advancements in Nuclear Instrumentation Measurement Methods and their Applications, France, 2009.

[5] G. Vayakis, S. Arshad, D. Delhom, A. Encheva, T. Giacomin, L. Jones, K.M. Patel, M. Pérez-Lasala, M. Portales, D. Prieto, F. Sartori, S. Simrock, J.A. Snipes, V.S. Udintsev, C. Watts, A. Winter, L. Zabeo, Development of the ITER magnetic diagnostic set and specification, Rev. Sci. Instrum. 83 (2012) 10D712.

[6] M. Kocan, I. Duran, S. Entler, G. Vayakis, J.M. Carmona, P. Gitton, J. Guirao, M. Gonzalez, S. Iglesias, Q. Pascual, G. Sandford, C. Vacas, M. Walsh, R. Walton, Final design of the ITER outer vessel steady-state magnetic sensors, Fusion Eng. Des. 123 (2017) 936-939.

[7] A. Gusarov, W. Leysen, M. Wuilpart, P. Mégret, Status and future developments of R \&D on fiber optics current sensor for ITER, Fusion Eng. Des. (2018) (in press).

[8] D.L. Brower, W.X. Ding, S.D. Terry, J.K. Anderson, T.M. Biewer, B.E. Chapman, D. Craig, C.B. Forest, S.C. Prager, J.S. Sarff, Laser polarimetric measurement of equilibrium and fluctuating magnetic fields in a reversed field pinch (invited), Rev. Sci. Instrum. 74 (2003) 1534-1540.

[9] Z.Y. Zou, H.Q. Liu, W.X. Ding, D.L. Brower, W.M. Li, T. Lan, Y.X. Jie, Effects of stray lights on Faraday rotation measurement for polarimeter-interferometer system on EAST, Rev. Sci. Instrum. 89 (2018) 013510

[10] J. Hao, P. Huang, X. Li, H. Yang, J. Yang, Polarization errors analysis of waveplate in Sagnac optical fiber current sensor, Proc. International Conference on Electronic and Mechanical Engineering and Information Technology, China, 2011.

[11] L. Wang, X. Xu, X. Liu, T. Zhang, J. Yan, Q. Ji, Modeling and simulation of polarization errors in reflective fiber optic current sensor, Opt. Eng. 50 (2011) 074402

[12] R.I. Laming, D.N. Payne, Electric current sensors employing spun highly birefringent optical fibers, J. Lightwave Technol. 7 (1986) 2084-2094. 
[13] Y.O. Barmenkov, F. Mendoza-Santoyo, Faraday plasma current sensor with compensation for reciprocal birefringence induced by mechanical perturbations, J. Appl. Res. Technol. 1 (2003) 157-163.

[14] P. Drexler, P. Fiala, Utilization of faraday mirror in fiber optic current sensors, Radioengineering 17 (2008) 101-107.

[15] M. Aerssens, A. Gusarov, B. Brichard, P. Mégret, V. Massaut, M. Wuilpart, Faraday effect based optical fiber current sensors for tokamaks, Proc. Conference on Advancements in Nuclear Instrumentation Measurement Methods and their Applications, Belgium, 2011.

[16] M. Aerssens, F. Descamps, A. Gusarov, P. Mégret, P. Moreau, M. Wuilpart, Influence of the optical fiber type on the performances of fiber-optics current sensor dedicated to plasma current measurement in ITER, Appl. Opt. 54 (2015) 5983-5991.

[17] M. Wuilpart, B. Vanus, A. Andrasan, A. Gusarov, P. Moreau, P. Mégret, Study of a fibre optics current sensor for the measurement of plasma current in ITER, Proc. Sixth European Workshop on Optical Fibre Sensors, Ireland, 2016.

[18] I.L. Lovchii, Numerical modelling and investigation of a polarimetric current transducer with a spun-type lightguide, J. Opt. Technol. 77 (2010) 755-761.

[19] H.S. Lassing, W.J. Mastop, A.F.G. van der Meer, A.A.M. Oomens, Plasma current measurements by Faraday rotation in a single-mode fiber, Appl. Opt. 26 (1987) 2456-2460.

[20] M.V. Andres, J.L. Cruz, M.A. Hernandez, Faraday effect in standard optical fibers: dispersion of the effective Verdet constant, Appl. Opt. 35 (1996) 922-927.

[23] F. Descamps, M. Aerssens, A. Gusarov, P. Mégret, V. Massaut, M. Wuilpart, Simulation of vibration-induced effect on plasma current measurement using a fiber optic current sensor, Opt. Express 22 (2014) 14666-14680.

[24] M. Wuilpart, M. Aerssens, A. Gusarov, P. Moreau, P. Megret, Plasma current measurement in thermonuclear fusion reactors using a photon-counting POTDR, IEEE Photonics Technol. Lett. (2017) 547-550.

[25] http://www.generalphotonics.com/index.php/product/frm-faraday-mirror/.

[26] M. Wuilpart, M. Tür, Polarization effects in optical fibers, in: L. Thévenaz (Ed.), Advanced Fiber Optics: Concepts and Technology, EPFL Press, 2011(Chapter 2).

[27] Lunainc.com, Measuring the Polarization Rotation Angle of a Faraday Rotator Mirror with the Optical Vector Analyzer, April 2014 [Online]. Available at: http:// lunainc.com/resource-library/measuring-polarization-rotation-angle-faradayrotator-mirror-optical-vector-analyzer (accessed 18.10.16). 